\title{
From absolute distinguishability to positive distinguishability
}

\author{
Zvika Brakerski and Oded Goldreich* \\ Department of Computer Science \\ Weizmann Institute of Science, Rehovot, IsRAEL.
}

April 9, 2009

\begin{abstract}
We study methods of converting algorithms that distinguish pairs of distributions with a gap that has an absolute value that is noticeable into corresponding algorithms in which the gap is always positive (and noticeable). Our focus is on designing algorithms that, in addition to the tested string, obtain a fixed number of samples from each distribution. Needless to say, such algorithms can not provide a very reliable guess for the sign of the original distinguishability gap, still we show that even guesses that are noticeably better than random are useful in this setting.
\end{abstract}

Keywords: Computational Indistinguishability, Statistical Indistinguishability.

${ }^{*}$ Partially supported by the Israel Science Foundation (grant No. 1041/08). 


\section{The problem and its solutions}

This work addresses a generic technical problem that arises in the context of trying to establish the computational indistinguishability of certain pairs of probability ensembles. The problem refers to the fact that computational (and also statistical) indistinguishability is defined in terms of the absolute difference between probabilities, whereas it is typically easier to manipulate the difference itself. Thus, we seek a method of converting a non-negligible absolute difference into a non-negligible difference; that is, we wish the difference itself (rather than its absolute value) to be positive.

\subsection{A motivational example}

Many security definitions are formulated by referring to two pairs of probability ensembles that are indexed by strings, and requiring that these pairs of probability ensembles are computationally indistinguishable (see, e.g., the definitions of computational zero-knowledge [2, Sec. 4.3.1.2] and secure two-party computation [3, Sec. 7.2]). Such a probability ensemble $\left\{Z_{\alpha}\right\}_{\alpha \in S}$ consists of (an infinite number of) "random variables" $Z_{\alpha}$ 's, which are each distributed over some finite set (related to its index, $\alpha$ ). Two such ensembles, $\left\{X_{\alpha}\right\}_{\alpha \in S}$ and $\left\{Y_{\alpha}\right\}_{\alpha \in S}$, are said to be computationally indistinguishable if for every probabilistic polynomial-time algorithm $D$ it holds that

$$
g_{D}(\alpha) \stackrel{\text { def }}{=}\left|\operatorname{Pr}\left[D\left(\alpha, X_{\alpha}\right)=1\right]-\operatorname{Pr}\left[D\left(\alpha, Y_{\alpha}\right)=1\right]\right|
$$

is negligible as a function of $|\alpha|$ (i.e., for every positive polynomial $p$ and all sufficiently long $\alpha$ 's the value of $g_{D}(\alpha)$ is upper bounded by $\left.1 / p(|\alpha|)\right)$.

The aforementioned formulation mandates that the value of $g_{D}(\alpha)$ is small for every $\alpha \in S$. A weaker requirement, which suffices in practice, is that it is infeasible to find $\alpha \in S$ for which the value of $g_{D}(\alpha)$ is not small. This requirement may be formulated as mandating that for every probabilistic polynomial-time algorithm $F$, representing a potential finder that given $1^{n}$ outputs an $n$-bit long string $\alpha \in S$, the expected value of $g_{D}(\alpha)$ (when defined as in Eq. (1)) is negligible (as a function of $n$ ); that is, $E\left[g_{D}\left(F\left(1^{n}\right)\right)\right]$ is negligible in $n$. This condition means that

$$
\sum_{\alpha} \operatorname{Pr}\left[F\left(1^{n}\right)=\alpha\right] \cdot\left|\operatorname{Pr}\left[D\left(\alpha, X_{\alpha}\right)=1\right]-\operatorname{Pr}\left[D\left(\alpha, Y_{\alpha}\right)=1\right]\right|
$$

is negligible as a function of $n$.

When trying to establish a condition as in Eq. (2) it is often easier to establish a corresponding condition in which the absolute value operator is dropped. Indeed, suppose that for every $F$ and $D$ as above it holds that

$$
\sum_{\alpha} \operatorname{Pr}\left[F\left(1^{n}\right)=\alpha\right] \cdot\left(\operatorname{Pr}\left[D\left(\alpha, X_{\alpha}\right)=1\right]-\operatorname{Pr}\left[D\left(\alpha, Y_{\alpha}\right)=1\right]\right)
$$

is negligible (as a function of $n$ ). Can we infer that Eq. (2) holds too?

In the case that both ensembles are polynomial-time sampleable, a positive answer is implicit in many works. Essentially, given a probabilistic polynomial-time algorithm $D$ such that Eq. (2) is not negligible, one derives a probabilistic polynomial-time algorithm $D^{\prime}$ such that Eq. (3) is not negligible by estimating the difference between $\operatorname{Pr}\left[D\left(\alpha, X_{\alpha}\right)=1\right]$ and $\operatorname{Pr}\left[D\left(\alpha, Y_{\alpha}\right)=1\right]$ and flipping $D$ 's output if the estimated difference is negative. Thus, the construction of $D^{\prime}$ depends also on $g_{D}$ (which determines the adequate level of approximation). In particular, the time complexity of $D^{\prime}$ is (polynomially) related to $g_{D}$. Our goal is to get rid of this dependency; in particular, we wish to avoid the aforementioned approximation. 


\subsection{A generic problem and one solution}

The generic problem we face is converting an algorithm $D$ that distinguishes $X_{\alpha}$ and $Y_{\alpha}$ (i.e., $\left|\operatorname{Pr}\left[D\left(\alpha, X_{\alpha}\right)=1\right]-\operatorname{Pr}\left[D\left(\alpha, Y_{\alpha}\right)=1\right]\right|$ is noticeable) into an algorithm $D^{\prime}$ that on input $\left(\alpha, X_{\alpha}\right)$ outputs 1 with probability that is noticeably higher than $\operatorname{Pr}\left[D\left(\alpha, Y_{\alpha}\right)=1\right]$. We stress that we wish this transformation to hold for every $\alpha$, whereas it may be that for some $\alpha$ 's the difference $\operatorname{Pr}\left[D\left(\alpha, X_{\alpha}\right)=1\right]-\operatorname{Pr}\left[D\left(\alpha, Y_{\alpha}\right)=1\right]$ is positive while for other $\alpha$ 's the difference is negative. Clearly, $D^{\prime}$ must know something about $X_{\alpha}$ and $Y_{\alpha}$ in order for this to be possible, and indeed we provide $D^{\prime}$ with samples taken from $X_{\alpha}$ and $Y_{\alpha}$ (or, actually, with algorithms for sampling these distributions).

Thus, the problem we face is actually the following one. We are given a probabilistic polynomialtime algorithm $D$ and sampling algorithms for two ensembles, $\left\{X_{\alpha}\right\}_{\alpha \in S}$ and $\left\{Y_{\alpha}\right\}_{\alpha \in S}$ (i.e., probabilistic polynomial-time algorithms $X$ and $Y$ such that on any input $\alpha$ it holds that $X_{\alpha} \equiv X(\alpha)$ and $\left.Y_{\alpha} \equiv Y(\alpha)\right)$. Our task is to construct a probabilistic polynomial-time algorithm $D^{\prime}$ such that for some function $\rho:(0,1] \rightarrow(0,1]$ it holds that

$$
\operatorname{Pr}\left[D^{\prime}\left(\alpha, X_{\alpha}\right)=1\right]-\operatorname{Pr}\left[D^{\prime}\left(\alpha, Y_{\alpha}\right)=1\right] \geq \rho\left(\left|\operatorname{Pr}\left[D\left(\alpha, X_{\alpha}\right)=1\right]-\operatorname{Pr}\left[D\left(\alpha, Y_{\alpha}\right)=1\right]\right|\right) .
$$

We stress that the r.h.s of Eq. (4) refers to the absolute difference between two probabilities, whereas the l.h.s refers to a corresponding difference that is not taken in absolute value and yet is required to be positive (whenever the former difference is positive).

We seek a universal transformation of $D$ into $D^{\prime}$, whereas this transformation may use a predetermined number of auxiliary samples of the two distributions. That is, the resulting algorithm $D^{\prime}$ is given as input a single sample that is drawn from one of two distributions (i.e., either from $X_{\alpha}$ or from $Y_{\alpha}$ ), but in addition it can obtain (a predetermined number of) samples from each of the two distributions. Like $D$, algorithm $D^{\prime}$ should distinguish the two cases (which correspond to the source of its input). We stress that we wish the complexity of $D^{\prime}$ (and specifically the number of auxiliary samples it obtains) to be independent of $g_{D}(\alpha)$. We note that such a transformation (of $D$ into $D^{\prime}$ ) may be useful also in other settings. One such generic example is provided by settings in which the notion of negligible probability being considered is significantly smaller than the reciprocal of the complexity of the distinguishers (e.g., consider polynomial-time distinguishers coupled with (sub-)exponentially small distinguishing gaps).

A simple transformation. One solution to the foregoing problem is to let $D^{\prime}$ estimate the sign of $\operatorname{Pr}\left[D\left(\alpha, X_{\alpha}\right)=1\right]-\operatorname{Pr}\left[D\left(\alpha, Y_{\alpha}\right)=1\right]$ by using a single sample of $X_{\alpha}$ and a single sample of $Y_{\alpha}$. (Although this estimate is quite poor, it can be shown to suffice.) Specifically, on input ( $\alpha$ and) $z$ (where $z$ is taken from either $X_{\alpha}$ or $Y_{\alpha}$ ), algorithm $D^{\prime}$ proceeds as follows:

1. Ignoring its ("main") input (i.e., $z$ ), algorithm $D^{\prime}$ generates a single sample $x$ of $X_{\alpha}$ and a single sample $y$ of $Y_{\alpha}$, and computes $\sigma \leftarrow D(\alpha, x)$ and $\tau \leftarrow D(\alpha, y)$;

2. If $\sigma>\tau$ then $D^{\prime}$ invokes $D$ on its input and outputs $D(\alpha, z)$.

If $\sigma<\tau$ then $D^{\prime}$ outputs $1-D(\alpha, z)$.

Otherwise (i.e., $\sigma=\tau$ ), algorithm $D^{\prime}$ outputs the outcome of a fair coin toss.

Indeed, we have assumed here (without loss of generality) that $D$ always outputs a Boolean value. Intuitively, $\sigma-\tau$ provides a probabilistic guess of the sign of $\operatorname{Pr}\left[D\left(\alpha, X_{\alpha}\right)=1\right]-\operatorname{Pr}\left[D\left(\alpha, Y_{\alpha}\right)=1\right]$, and using this guess in the obvious manner yields the desired result. 
Proposition 1.1 Let $D$ and $D^{\prime}$ be as above. Then,

$$
\operatorname{Pr}\left[D^{\prime}\left(\alpha, X_{\alpha}\right)=1\right]-\operatorname{Pr}\left[D^{\prime}\left(\alpha, Y_{\alpha}\right)=1\right]=\left(\left|\operatorname{Pr}\left[D\left(\alpha, X_{\alpha}\right)=1\right]-\operatorname{Pr}\left[D\left(\alpha, Y_{\alpha}\right)=1\right]\right|\right)^{2} .
$$

Proof: For the analysis of the performance of $D^{\prime}$, we consider an algorithm $D^{\prime \prime}$, which may output any number in $[0,1]$, such that

$$
D^{\prime \prime}(\alpha, z) \stackrel{\text { def }}{=} \frac{1}{2} \cdot\left(1+\operatorname{sign}\left(D\left(\alpha, X_{\alpha}\right)-D\left(\alpha, Y_{\alpha}\right)\right) \cdot(-1)^{D(\alpha, z)+1}\right)
$$

where $\operatorname{sign}(r)=1$ if $r>0$ (resp., $\operatorname{sign}(r)=-1$ if $r<0$ ), and $\operatorname{sign}(0)=0$. Recall that in Step 2 of $D^{\prime}(\alpha, z)$, the output is set to $D(\alpha, z)$ if $\sigma>\tau$, to $1-D(\alpha, z)$ if $\sigma<\tau$, and is random if $\sigma=\tau$. Using $D(\alpha, z) \in\{0,1\}$ and assuming $\sigma \neq \tau$, the output of $D^{\prime}(\alpha, z)$ can be written as $\left(1+\operatorname{sign}(\sigma-\tau) \cdot(-1)^{D(\alpha, z)+1}\right) / 2$. Thus, $D^{\prime}(\alpha, z)$ outputs 1 with probability $D^{\prime \prime}(\alpha, z)$, and it suffices to evaluate

$$
\mathrm{E}\left[D^{\prime \prime}\left(\alpha, X_{\alpha}\right)\right]-\mathrm{E}\left[D^{\prime \prime}\left(\alpha, Y_{\alpha}\right)\right]=\operatorname{Pr}\left[D^{\prime}\left(\alpha, X_{\alpha}\right)=1\right]-\operatorname{Pr}\left[D^{\prime}\left(\alpha, Y_{\alpha}\right)=1\right] .
$$

Denoting $p=\operatorname{Pr}\left[D\left(\alpha, X_{\alpha}\right)=1\right]$ and $q=\operatorname{Pr}\left[D\left(\alpha, Y_{\alpha}\right)=1\right]$ (and using $X_{\alpha}^{\prime}$ and $Y_{\alpha}^{\prime}$ to denote independent copies of $X_{\alpha}$ and $Y_{\alpha}$ ), we evaluate Eq. (6) as follows.

$$
\begin{aligned}
g_{D^{\prime \prime}}(\alpha) \stackrel{\text { def }}{=} & \mathrm{E}\left[D^{\prime \prime}\left(\alpha, X_{\alpha}\right)\right]-\mathrm{E}\left[D^{\prime \prime}\left(\alpha, Y_{\alpha}\right)\right] \\
= & \frac{1}{2} \cdot \mathrm{E}\left[1+\operatorname{sign}\left(D\left(\alpha, X_{\alpha}^{\prime}\right)-D\left(\alpha, Y_{\alpha}^{\prime}\right)\right) \cdot(-1)^{D\left(\alpha, X_{\alpha}\right)+1}\right] \\
& -\frac{1}{2} \cdot \mathrm{E}\left[1+\operatorname{sign}\left(D\left(\alpha, X_{\alpha}^{\prime}\right)-D\left(\alpha, Y_{\alpha}^{\prime}\right)\right) \cdot(-1)^{D\left(\alpha, Y_{\alpha}\right)+1}\right] \\
= & \frac{1}{2} \cdot \mathrm{E}\left[\operatorname{sign}\left(D\left(\alpha, X_{\alpha}^{\prime}\right)-D\left(\alpha, Y_{\alpha}^{\prime}\right)\right)\right] \cdot \mathrm{E}\left[(-1)^{D\left(\alpha, X_{\alpha}\right)+1}-(-1)^{D\left(\alpha, Y_{\alpha}\right)+1}\right]
\end{aligned}
$$

Using $\mathrm{E}\left[(-1)^{D\left(\alpha, X_{\alpha}\right)+1}\right]=p-(1-p)=2 p-1$ and $\mathrm{E}\left[(-1)^{D\left(\alpha, Y_{\alpha}\right)+1}\right]=2 q-1$, we get

$$
\begin{aligned}
g_{D^{\prime \prime}}(\alpha) & =(p-q) \cdot \mathrm{E}\left[\operatorname{sign}\left(D\left(\alpha, X_{\alpha}\right)-D\left(\alpha, Y_{\alpha}\right)\right)\right] \\
& =(p-q) \cdot\left(\operatorname{Pr}\left[D\left(\alpha, X_{\alpha}\right)>D\left(\alpha, Y_{\alpha}\right)\right]-\operatorname{Pr}\left[D\left(\alpha, X_{\alpha}\right)<D\left(\alpha, Y_{\alpha}\right)\right]\right) \\
& =(p-q) \cdot(p \cdot(1-q)-(1-p) \cdot q)
\end{aligned}
$$

which equals $(p-q)^{2}$.

\subsection{Other transformations}

Two natural questions arise:

1. Is the foregoing construction of $D^{\prime}$ optimal (with respect to all constructions that use a single auxiliary sample from each of the two distributions)?

2. Can we do better if we obtain $k$ auxiliary samples from each of the two distributions (rather than one auxiliary sample from each of the two distributions)? How good can such a construction be?

Before answering these questions we note that no construction (which is given a single test sample from an unknown distribution) can outperform the variation distance between the tested distributions, (i.e., $|p-q|$, where $p=\operatorname{Pr}\left[D\left(\alpha, X_{\alpha}\right)=1\right]$ and $\left.q=\operatorname{Pr}\left[D\left(\alpha, Y_{\alpha}\right)=1\right]\right)$. We answer the above questions as follows. 
Main Result (informal). For every $k \geq 1$, the best construction that uses $k$ auxiliary samples from each of the two distributions is the one that rules analogously to Eq. (5), when applying the sign function to the difference between the average value of $D$ in the two cases. Such a procedure yields a gap that equals the minimum of $\Omega(\sqrt{k}) \cdot(p-q)^{2}$ and $\left(1-\epsilon_{p, q}(k)\right) \cdot|p-q|$, where $\epsilon_{p, q}(k)=$ $\exp \left(-\Omega\left((p-q)^{2} \cdot k\right)\right)$.

We stress that the above result holds both in the computational setting and in the information theoretic setting.

\section{The actual treatment}

Let $X$ and $Y$ be $0-1$ random variables (representing $D\left(\alpha, X_{\alpha}\right)$ and $D\left(\alpha, Y_{\alpha}\right)$, respectively), and let $X_{i}$ 's (resp., $Y_{i}$ 's) be independent copies of $X$ (resp., $Y$ ) representing additional samples available to us. We seek a randomized process $\Pi:\{0,1\}^{2 k+1} \rightarrow\{0,1\}$ such that

$$
\mathrm{E}\left[\Pi\left(X_{1}, \ldots, X_{k}, Y_{1}, \ldots, Y_{k}, X\right)\right]-\mathrm{E}\left[\Pi\left(X_{1}, \ldots, X_{k}, Y_{1}, \ldots, Y_{k}, Y\right)\right]
$$

is maximized (as a function of $\delta=|\mathrm{E}[X]-\mathrm{E}[Y]|$, when maximizing over all possible 0-1 random variables $X$ and $Y$ that are at statistical distance $\delta)$. Indeed, the probability that $\Pi\left(a_{1}, \ldots, a_{k}, b_{1}, \ldots, b_{k}, c\right)=$ 1 is determined by a function $f:\{0,1\}^{2 k+1} \rightarrow[0,1]$ such that

$$
\operatorname{Pr}\left[\Pi\left(a_{1}, \ldots, a_{k}, b_{1}, \ldots, b_{k}, c\right)=1\right]=f\left(a_{1}, \ldots, a_{k}, b_{1}, \ldots, b_{k}, c\right)
$$

Thus, it suffices to seek such a function $f$ that maximizes

$$
\mathrm{E}\left[f\left(X_{1}, \ldots, X_{k}, Y_{1}, \ldots, Y_{k}, X\right)\right]-\mathrm{E}\left[f\left(X_{1}, \ldots, X_{k}, Y_{1}, \ldots, Y_{k}, Y\right)\right]
$$

(as a function of $\delta=|\mathrm{E}[X]-\mathrm{E}[Y]|$ ).

Let us formally define a more general optimization problem. For a function $f:\{0,1\}^{2 k+1} \rightarrow$ $[0,1]$ and a pair $(p, q) \in[0,1]$, we denote by $\mathcal{V}_{(p, q)}(f)$ the value of Eq. (8), when $X$ and $Y$ satisfy $p=\mathrm{E}[X]$ and $q=\mathrm{E}[Y]$. Now, for any (possibly infinite) set (or class) of pairs in $[0,1]$, denoted $\mathcal{C}$, and any function $f:\{0,1\}^{2 k+1} \rightarrow[0,1]$, we denote $\mathcal{V}_{\mathcal{C}}(f) \stackrel{\text { def }}{=} \min _{(p, q) \in \mathcal{C}}\left\{\mathcal{V}_{(p, q)}(f)\right\}$. We seek a function $f$ for which $\mathcal{V}_{\mathcal{C}}(f)$ is maximal.

Overview. First, we will show that, without loss of generality, the function $f\left(x_{1}, \ldots, x_{k}, y_{1}, \ldots, y_{k}, z\right)$ may only depend on $s \stackrel{\text { def }}{=} \sum_{i \in[k]} x_{i}, t \stackrel{\text { def }}{=} \sum_{i \in[k]} y_{i}$ and $z$, and furthermore that it can take a specific canonical form (see Section 2.1). Next, in Section 2.2, we will show that in all natural cases (i.e., for "symmertic" classes) the canonical form can be further simplified to depend only on sign $(s-t)$ and $z$. Actually, this will yield a single optimal function. Lastly, in Section 2.3, we will analyze the performance of this function.

\subsection{Canonical functions}

We will first show that it suffices to consider functions $f$ of the form

$$
f\left(a_{1}, \ldots, a_{k}, b_{1}, \ldots, b_{k}, c\right)=\frac{1+g\left(\sum_{i \in[k]} a_{i}, \sum_{i \in[k]} b_{i}\right) \cdot(-1)^{c}}{2}
$$

where $g: \mathbb{N}^{2} \rightarrow[-1,+1]$. We call such an $f$ canonical. Note that the normalization (i.e., shifting by 1 and dividing by 2 ) is used to map $[-1,+1]$ to $[0,1]$. (Note that an additive shift of $f$ leaves the value of Eq. (8) intact, whereas multiplying $f$ by any factor has the same effect on the value of Eq. (8).) 
Definition 2.1 (dominating strategies) We say that $f^{\prime}$ dominates $f$ (w.r.t $\mathcal{C}$ ) if for every $(p, q) \in \mathcal{C}$ it holds that $\mathcal{V}_{(p, q)}\left(f^{\prime}\right) \geq \mathcal{V}_{(p, q)}(f)$.

Proposition 2.2 (strong optimality): For every $\mathcal{C}$ and every $f:\{0,1\}^{2 k+1} \rightarrow[-1,+1]$ there exists a canonical function that dominates $f$.

Proof: Given any function $f$, we consider the function $f^{\prime}$ such that for every $a, b \in\{0,1, \ldots, k\}$ and $c \in\{0,1\}$, the value $f^{\prime}(a, b, c)$ equals the average of $f\left(a_{1}, \ldots, a_{k}, b_{1}, \ldots, b_{k}, c\right)$ taken over all $\left(a_{1}, \ldots, a_{k}\right),\left(b_{1}, \ldots, b_{k}\right) \in\{0,1\}^{k}$ that satisfy $\sum_{i \in[k]} a_{i}=a$ and $\sum_{i \in[k]} b_{i}=b$. Then, for every $(p, q)$, we have $\mathcal{V}_{(p, q)}\left(f^{\prime}\right)=\mathcal{V}_{(p, q)}(f)$. Note that the value of $f^{\prime}$ at any $(a, b)$ and $c \in\{0,1\}$ can be written as

$$
\begin{aligned}
& \frac{1+(-1)^{c}}{2} \cdot f^{\prime}(a, b, 0)+\frac{1-(-1)^{c}}{2} \cdot f^{\prime}(a, b, 1) \\
& =\frac{1}{2} \cdot\left(f^{\prime}(a, b, 0)+f^{\prime}(a, b, 1)\right)+\frac{(-1)^{c}}{2} \cdot\left(f^{\prime}(a, b, 0)-f^{\prime}(a, b, 1)\right) \\
& =g_{0}(a, b)+g_{1}(a, b) \cdot(-1)^{c}
\end{aligned}
$$

where $g_{0}(a, b)=\left(f^{\prime}(a, b, 0)+f^{\prime}(a, b, 1)\right) / 2$ and $g_{1}(a, b)=\left(f^{\prime}(a, b, 0)-f^{\prime}(a, b, 1)\right) / 2$. Note that $g_{1}(a, b) \in[-0.5,+0.5]$ and that replacing $g_{0}(a, b)$ by 0.5 does not change the value of $\mathcal{V}_{(p, q)}\left(f^{\prime}\right)$. Thus, setting $f^{\prime \prime}(a, b, c)=\left(1+2 g_{1}(a, b) \cdot(-1)^{c}\right) / 2$, we obtain a canonical function that dominates $f$ (because $\left.\mathcal{V}_{(p, q)}\left(f^{\prime \prime}\right)=\mathcal{V}_{(p, q)}\left(f^{\prime}\right)=\mathcal{V}_{(p, q)}(f)\right)$.

Conclusion and Notation. At this point we can limit our search for good functions (i.e., functions that maximize Eq. (8)) to canonical functions. That is, for every function $g: \mathbb{N}^{2} \times\{0,1\} \rightarrow$ $[-1,+1]$ and every $k \in \mathbb{N}$, we define $f_{g}^{(k)}$ as in Eq. (9), and consider the value $\mathcal{V}_{(p, q)}\left(f_{g}^{(k)}\right)$. To estimate $\mathcal{V}_{(p, q)}\left(f_{g}^{(k)}\right)$, we let $X$ and $Y$ be $0-1$ random variables with $\mathrm{E}[X]=p$ and $\mathrm{E}[Y]=q$ and get

$$
\mathcal{V}_{(p, q)}\left(f_{g}^{(k)}\right)=\frac{1}{2} \cdot \mathrm{E}\left[g\left(\sum_{i \in[k]} X_{i}, \sum_{i \in[k]} Y_{i}\right) \cdot(-1)^{X}\right]-\frac{1}{2} \cdot \mathrm{E}\left[g\left(\sum_{i \in[k]} X_{i}, \sum_{i \in[k]} Y_{i}\right) \cdot(-1)^{Y}\right]
$$

Using the independence of $X, Y$ and the $X_{i}$ 's and $Y_{i}$ 's, we rewrite Eq. (10) as

$$
\mathcal{V}_{(p, q)}\left(f_{g}^{(k)}\right)=\frac{1}{2} \cdot \mathrm{E}\left[g\left(\sum_{i \in[k]} X_{i}, \sum_{i \in[k]} Y_{i}\right)\right] \cdot \mathrm{E}\left[(-1)^{X}-(-1)^{Y}\right] .
$$

Recalling that $\mathrm{E}\left[(-1)^{X}\right]=(1-p)-p=1-2 p$ and $\mathrm{E}\left[(-1)^{Y}\right]=1-2 q$, we get $\mathrm{E}\left[(-1)^{X}-(-1)^{Y}\right]=$ $2(q-p)$ and so

$$
\mathcal{V}_{(p, q)}\left(f_{g}^{(k)}\right)=(q-p) \cdot \mathrm{E}\left[g\left(X^{\prime}, Y^{\prime}\right)\right]
$$

where $X^{\prime}=\sum_{i \in[k]} X_{i}$ and $Y^{\prime}=\sum_{i \in[k]} Y_{i}$. Denoting $B(p, i, k)=\left(\begin{array}{c}k \\ i\end{array}\right) \cdot p^{i} \cdot(1-p)^{k-i}$, we get

$$
\mathcal{V}_{(p, q)}\left(f_{g}^{(k)}\right)=(q-p) \cdot \sum_{i, j \in\{0,1, \ldots, k\}} B(p, i, k) \cdot B(q, j, k) \cdot g(i, j)
$$




\section{$2.2 \quad$ Symmetric classes}

We focus on symmetric classes of pairs, where $\mathcal{C}$ is symmetric if for every $(p, q) \in \mathcal{C}$ it also holds that $(q, p) \in \mathcal{C}$. In contrast, if $\mathcal{C}$ contains only pairs $(p, q)$ such that $p>q$, then we may set $k=0$ and use the identity function (because $\mathrm{E}[X]-\mathrm{E}[Y]=p-q=\operatorname{StatDiff}(X, Y)$ ). We show that, for symmetric classes, the "sign of the difference" function (i.e., $\operatorname{sd}(a, b)=\operatorname{sign}(b-a) \in\{-1,0,+1\}$ ) is optimal as a function $g$.

Proposition 2.3 (optimality): For every symmetric $\mathcal{C}$ and every $k \in \mathbb{N}$ and $g: \mathbb{N}^{2} \rightarrow[-1,+1]$, it holds that $\mathcal{V}_{\mathcal{C}}\left(f_{\text {sd }}^{(k)}\right) \geq \mathcal{V}_{\mathcal{C}}\left(f_{g}^{(k)}\right)$, where $\operatorname{sd}(a, b)=\operatorname{sign}(b-a)$.

Recall that $\operatorname{sign}(d)=-1$ if $d<0$ (resp., $\operatorname{sign}(d)=1$ if $d>0$ ), and $\operatorname{sign}(0)=0$.

Proof: Let $(p, q) \in \mathcal{C}$ be such that $\mathcal{V}_{(p, q)}\left(f_{\text {sd }}^{(k)}\right)=\mathcal{V}_{\mathcal{C}}\left(f_{\text {sd }}^{(k)}\right)$. Then, $\mathcal{V}_{\mathcal{C}}\left(f_{g}^{(k)}\right) \leq\left(\mathcal{V}_{(p, q)}\left(f_{g}^{(k)}\right)+\right.$ $\left.\mathcal{V}_{(q, p)}\left(f_{g}^{(k)}\right)\right) / 2$ (by definition of $\mathcal{V}_{\mathcal{C}}\left(f_{g}^{(k)}\right)$ and the fact that $(q, p) \in \mathcal{C}$ [which follows by the symmetry of $\mathcal{C}]$ ), whereas $\mathcal{V}_{\mathcal{C}}\left(f_{\text {sd }}^{(k)}\right) \geq \mathcal{V}_{(p, q)}\left(f_{\text {sd }}^{(k)}\right)$ (by the choice of $\left.(p, q) \in \mathcal{C}\right)$. Also note that $\mathcal{V}_{(p, q)}\left(f_{\mathrm{sd}}^{(k)}\right)=$ $\mathcal{V}_{(q, p)}\left(f_{\mathbf{s d}}^{(k)}\right)$ (by the invariance of the function $f_{\text {sd }}^{(k)}$ under of this switch, as seen in Eq. (12)). Thus, it suffices to show that

$$
\mathcal{V}_{(p, q)}\left(f_{\text {sd }}^{(k)}\right)+\mathcal{V}_{(q, p)}\left(f_{\text {sd }}^{(k)}\right) \geq \mathcal{V}_{(p, q)}\left(f_{g}^{(k)}\right)+\mathcal{V}_{(q, p)}\left(f_{g}^{(k)}\right)
$$

For every $a, b \in\{0,1, \ldots, k\}$, we shall show that replacing $g(a, b)$ by $\operatorname{sign}(b-a)$ may only increase $\mathcal{V}_{(p, q)}\left(f_{g}^{(k)}\right)+\mathcal{V}_{(q, p)}\left(f_{g}^{(k)}\right)$. Let us start by recalling Eq. (13), which yields

$$
\begin{aligned}
\mathcal{V}_{(p, q)}\left(f_{g}^{(k)}\right)+\mathcal{V}_{(q, p)}\left(f_{g}^{(k)}\right)= & (q-p) \cdot \sum_{i, j \in\{0,1, \ldots, k\}} B(p, i, k) B(q, j, k) \cdot g(i, j) \\
& +(p-q) \cdot \sum_{i, j \in\{0,1, \ldots, k\}} B(q, i, k) B(p, j, k) \cdot g(i, j) \\
= & (q-p) \cdot \sum_{i, j \in\{0,1, \ldots, k\}}[B(p, i, k) B(q, j, k)-B(q, i, k) B(p, j, k)] \cdot g(i, j) .
\end{aligned}
$$

Clearly, for $i=j$ we have $B(p, i, k) B(q, j, k)=B(q, i, k) B(p, j, k)$. For $i<j$ (resp., $j<i$ ), it holds that $B(p, i, k) B(q, j, k)>B(q, i, k) B(p, j, k)$ if and only if $p<q$ (resp., $q<p)$. The latter claim seems self-evident, yet we provide a detailed proof next (for the case $p, q \in(0,1)$ ).

$$
\begin{aligned}
B(p, i, k) B(q, j, k) & =\left(\begin{array}{c}
k \\
i
\end{array}\right) \cdot p^{i} \cdot(1-p)^{k-i} \cdot\left(\begin{array}{c}
k \\
j
\end{array}\right) \cdot q^{j} \cdot(1-q)^{k-j} \\
& =\left(\begin{array}{c}
k \\
i
\end{array}\right) \cdot(1-p)^{k} \cdot\left(\begin{array}{c}
k \\
j
\end{array}\right) \cdot(1-q)^{k} \cdot(p /(1-p))^{i} \cdot(q /(1-q))^{j}
\end{aligned}
$$

Thus, $\frac{B(p, i, k) B(q, j, k)}{B(q, i, k) B(p, j, k)}$ equals

$$
\frac{(p /(1-p))^{i} \cdot(q /(1-q))^{j}}{(q /(1-q))^{i} \cdot(p /(1-p))^{j}}=\frac{(q /(1-q))^{j-i}}{(p /(1-p))^{j-i}}
$$

Note that we have $p<q$ iff $(p /(1-p))<(q /(1-q))$, and so $p<q$ iff $(p /(1-p))^{j-i}<(q /(1-q))^{j-i}$. It follows that $p<q$ iff $B(p, i, k) B(q, j, k)>B(q, i, k) B(p, j, k)$. 
Recall that for $i<j$, it holds that $B(p, i, k) B(q, j, k)-B(q, i, k) B(p, j, k)>0$ if and only if $q>p$. Thus, in this case, we maximize

$$
(q-p) \cdot[B(p, i, k) B(q, j, k)-B(q, i, k) B(p, j, k)] \cdot g(i, j)
$$

by setting $g(i, j)=1$ (because the first two factors have the same sign). Similarly, for $j>i$, it holds that $B(p, i, k) B(q, j, k)-B(q, i, k) B(p, j, k)>0$ if and only if $q<p$, and so the maximization requires $g(i, j)=-1$. Indeed, for $i=j$, any setting of $g(i, j)$ will do. Thus, an optimal setting of $g(i, j)$ is $\operatorname{sign}(j-i)$, which equals $\operatorname{sd}(i, j)$. The claim follows.

\subsection{The performance of the function $f_{\mathrm{sd}}^{(k)}$}

We now turn to evaluating the performance of the optimal function; that is, we evaluate $\mathcal{V}_{(p, q)}\left(f_{\mathbf{s d}}^{(k)}\right)$. Recall that

$$
\begin{aligned}
\mathcal{V}_{(p, q)}\left(f_{\mathrm{sd}}^{(k)}\right) & =(q-p) \cdot \sum_{i, j \in\{0,1, \ldots, k\}} B(p, i, k) B(q, j, k) \cdot \operatorname{sd}(i, j) \\
& =(p-q) \cdot \sum_{i, j \in\{0,1, \ldots, k\}} B(p, i, k) B(q, j, k) \cdot \operatorname{sign}(i-j)
\end{aligned}
$$

which yields $\mathcal{V}_{(p, q)}\left(f_{\text {sd }}^{(k)}\right)=(p-q) \cdot v_{p, q}$, where

$$
v_{p, q} \stackrel{\text { def }}{=} \mathrm{E}\left[\operatorname{sign}\left(\sum_{i \in[k]} X_{i}-\sum_{i \in[k]} Y_{i}\right)\right]
$$

such that the $X_{i}$ 's (resp., $Y_{i}$ 's) are 0-1 i.i.d with expectation $p$ (resp., $q$ ). Letting $T_{i}=X_{i}-Y_{i}$, we rewrite Eq. (16) as $\mathrm{E}\left[\operatorname{sign}\left(\sum_{i \in[k]} T_{i}\right)\right]$, which equals

$$
\operatorname{Pr}\left[\sum_{i \in[k]} T_{i}>0\right]-\operatorname{Pr}\left[\sum_{i \in[k]} T_{i}<0\right] .
$$

Note that $\mathrm{E}\left[T_{i}\right]=p-q$ and $\operatorname{Var}\left[T_{i}\right]=p(1-p)+q(1-q)$.

The cases of $k=1$ and $k=2$. For small $k$, we can write explicit expressions for Eq. (17); for example, for $k=1$ Eq. (17) yields $\operatorname{Pr}\left[T_{1}>0\right]-\operatorname{Pr}\left[T_{1}<0\right]=p(1-q)-q(1-p)=p-q$, and so $\mathcal{V}_{(p, q)}\left(f_{\text {sd }}^{(1)}\right)=(p-q)^{2}$. For $k=2$, we have

$$
\begin{aligned}
\operatorname{Pr}\left[T_{1}+T_{2}>0\right]-\operatorname{Pr}\left[T_{1}+T_{2}<0\right]= & \operatorname{Pr}\left[T_{1}+T_{2}=2\right]+2 \operatorname{Pr}\left[T_{1}=1 \wedge T_{2}=0\right] \\
& -\left(\operatorname{Pr}\left[T_{1}+T_{2}=-2\right]+2 \operatorname{Pr}\left[T_{1}=-1 \wedge T_{2}=0\right]\right) \\
= & p^{2}(1-q)^{2}+2 p(1-q)(p q+(1-p)(1-q)) \\
& -\left(q^{2}(1-p)^{2}+2 q(1-p)(p q+(1-p)(1-q))\right) \\
= & (1+(1-p)(1-q)+p q) \cdot(p-q)
\end{aligned}
$$

and so $\mathcal{V}_{(p, q)}\left(f_{\text {sd }}^{(2)}\right)=(1+(1-p)(1-q)+p q) \cdot(p-q)^{2}$ (see alternative proof following Proposition 2.4). Thus, the improvement of the case of $k=2$ over the case of $k=1$ is a factor of $(1+(1-p)(1-q)+p q)$, which is greater than 1 unless $\{p, q\}=\{0,1\}$ (where a single sample is as good as $k$ samples, for any $k>1)$. 
The general case of $k>1$. We now turn to a general analysis of Eq. (17) (and $\left.\mathcal{V}_{(p, q)}\left(f_{\mathrm{sd}}^{(k)}\right)\right)$. Specifically, we consider the increase in the value of Eq. (17) when going from $k$ to $k+1$; that is, we define

$$
\Delta_{(p, q)}(k) \stackrel{\text { def }}{=} \mathrm{E}\left[\operatorname{sign}\left(\sum_{i \in[k+1]} T_{i}\right)\right]-\mathrm{E}\left[\operatorname{sign}\left(\sum_{i \in[k]} T_{i}\right)\right]
$$

and note that $\mathcal{V}_{(p, q)}\left(f_{\mathrm{sd}}^{(k+1)}\right)=\mathcal{V}_{(p, q)}\left(f_{\mathrm{sd}}^{(k)}\right)+(p-q) \cdot \Delta_{(p, q)}(k)$.

Proposition 2.4 (the growth of $\mathcal{V}_{(p, q)}\left(f_{\mathbf{s d}}^{(k)}\right)$ as a function of $k$ ): For every $k \geq 1$, it holds that $\Delta_{(p, q)}(k)=(p-q) \cdot \operatorname{Pr}\left[S_{k}=0\right]$, where $S_{k} \stackrel{\text { def }}{=} \sum_{i \in[k]} T_{i}$.

It follows that $\mathcal{V}_{(p, q)}\left(f_{\mathrm{sd}}^{(k+1)}\right)=\mathcal{V}_{(p, q)}\left(f_{\mathrm{sd}}^{(k)}\right)+(p-q)^{2} \cdot \operatorname{Pr}\left[S_{k}=0\right]$, and so $\mathcal{V}_{(p, q)}\left(f_{\mathrm{sd}}^{(k+1)}\right) \geq \mathcal{V}_{(p, q)}\left(f_{\mathrm{sd}}^{(k)}\right)$, where equality holds if and only if $\{p, q\}=\{0,1\}$ (when ignoring the case of $p=q$ ). Proposition 2.4 can also be used to re-establish $\mathcal{V}_{(p, q)}\left(f_{\text {sd }}^{(2)}\right)=(1+p q+(1-p)(1-q)) \cdot(p-q)^{2}$, since $\mathcal{V}_{(p, q)}\left(f_{\text {sd }}^{(1)}\right)=$ $(p-q)^{2}$ and $\operatorname{Pr}\left[S_{1}=0\right]=p q+(1-p)(1-q)$.

Proof: Starting with Eq. (18), we have

$$
\begin{aligned}
\Delta_{(p, q)}(k)= & \mathrm{E}\left[\operatorname{sign}\left(S_{k}+T_{k+1}\right)\right]-\mathrm{E}\left[\operatorname{sign}\left(S_{k}\right)\right] \\
= & \sum_{s \in\{-1,0,1\}} \operatorname{Pr}\left[S_{k}=s\right] \cdot \mathrm{E}\left[\operatorname{sign}\left(s+T_{k+1}\right)-\operatorname{sign}(s)\right] \\
= & \operatorname{Pr}\left[S_{k}=0\right] \cdot\left(\operatorname{Pr}\left[T_{k+1}=1\right]-\operatorname{Pr}\left[T_{k+1}=-1\right]\right) \\
& +\operatorname{Pr}\left[S_{k}=-1\right] \cdot \operatorname{Pr}\left[T_{k+1}=1\right]-\operatorname{Pr}\left[S_{k}=1\right] \cdot \operatorname{Pr}\left[T_{k+1}=-1\right]
\end{aligned}
$$

By symmetry (e.g., consider the case of $k=1$ ), it is rather self-evident that $\operatorname{Pr}\left[S_{k}=-1\right] \cdot \operatorname{Pr}\left[T_{k+1}=\right.$ 1] $=\operatorname{Pr}\left[S_{k}=1\right] \cdot \operatorname{Pr}\left[T_{k+1}=-1\right]$, yet we provide a detailed proof next.

$$
\begin{aligned}
\operatorname{Pr}\left[S_{k}=-1\right] \cdot \operatorname{Pr}\left[T_{k+1}=1\right] & =p(1-q) \cdot \sum_{j=1}^{k} B(p, j-1, k) B(q, j, k) \\
& =p(1-q) \cdot \sum_{j=1}^{k}\left(\begin{array}{c}
k \\
j-1
\end{array}\right) p^{j-1}(1-p)^{k-j+1}\left(\begin{array}{c}
k \\
j
\end{array}\right) q^{j}(1-q)^{k-j} \\
& =\sum_{j=1}^{k}\left(\begin{array}{c}
k \\
j-1
\end{array}\right) p^{j}(1-p)^{k+1-j}\left(\begin{array}{c}
k \\
j
\end{array}\right) q^{j}(1-q)^{k-j+1} \\
& =(1-p) q \sum_{j=1}^{k}\left(\begin{array}{c}
k \\
j-1
\end{array}\right) p^{j}(1-p)^{k-j}\left(\begin{array}{c}
k \\
j
\end{array}\right) q^{j-1}(1-q)^{k-j+1} \\
& =(1-p) q \cdot \sum_{j=1}^{k} B(p, j, k) B(q, j-1, k) \\
& =\operatorname{Pr}\left[S_{k}=1\right] \cdot \operatorname{Pr}\left[T_{k+1}=-1\right]
\end{aligned}
$$

Hence, $\Delta_{(p, q)}(k)=\operatorname{Pr}\left[S_{k}=0\right] \cdot\left(\operatorname{Pr}\left[T_{k+1}=1\right]-\operatorname{Pr}\left[T_{k+1}=-1\right]\right)$, and the claim follows (because $\left.\operatorname{Pr}\left[T_{k+1}=1\right]-\operatorname{Pr}\left[T_{k+1}=-1\right]=p-q\right)$. 
Proposition 2.4 yields another expression for $\mathcal{V}_{(p, q)}\left(f_{\text {sd }}^{(k)}\right)$ :

$$
\begin{aligned}
\mathcal{V}_{(p, q)}\left(f_{\text {sd }}^{(k)}\right) & =\mathcal{V}_{(p, q)}\left(f_{\mathbf{s d}}^{(1)}\right)+(p-q) \cdot \sum_{\ell=1}^{k-1} \Delta_{(p, q)}(\ell) \\
& =(p-q)^{2}+(p-q)^{2} \cdot \sum_{\ell=1}^{k-1} \operatorname{Pr}\left[S_{\ell}=0\right]
\end{aligned}
$$

Note that for $\{p, q\}=\{0,1\}$ this expression (i.e., Eq. (20)) equals 1 (for any $k \geq 1$ ), whereas for $p=q$ it equals 0 . In all other cases (i.e., $0<(p-q)^{2}<1$ ) Eq. (20) grows with $k$. Using $\operatorname{Pr}\left[S_{\ell}=0\right]=\sum_{j=0}^{\ell} B(p, j, \ell) B(q, j, \ell)$, we get

$$
\mathcal{V}_{(p, q)}\left(f_{\text {sd }}^{(k)}\right)=(p-q)^{2}+(p-q)^{2} \cdot \sum_{\ell=1}^{k-1} \sum_{j=0}^{\ell}\left(\begin{array}{l}
\ell \\
j
\end{array}\right)^{2}(p q)^{j}((1-p)(1-q))^{\ell-j}
$$

In the special case of $p=0$, Eq. (21) yields

$$
\begin{aligned}
\mathcal{V}_{(0, q)}\left(f_{\mathbf{s d}}^{(k)}\right) & =q^{2}+q^{2} \cdot \sum_{\ell=1}^{k-1}(1-q)^{\ell} \\
& =q^{2}+q \cdot\left((1-q)-(1-q)^{k}\right)
\end{aligned}
$$

which converges to $q=|p-q|$ when $k \rightarrow \infty$. Similarly, $\mathcal{V}_{(1, q)}\left(f_{\text {sd }}^{(k)}\right)$ converges to $1-q=|p-q|$ (where $p=1$ ). Note that in these cases convergence occurs with $k \gg|p-q|^{-1}$. As we shall see next, in the other cases (i.e., $p, q \in(0,1))$, convergence occurs with $k \gg|p-q|^{-2}$. We note that the constants in the approximation given next depend on the distance of $p$ and $q$ from the boundaries of $(0,1)$; that is, these constants depends on $\min (p, q, 1-p, 1-q)$.

Proposition 2.5 (the approximate value of $\left.\mathcal{V}_{(p, q)}\left(f_{\text {sd }}^{(k)}\right)\right)$ : For any fixed $p, q \in(0,1)$ and every $k>2$, it holds that $\mathcal{V}_{(p, q)}\left(f_{\mathrm{sd}}^{(k)}\right)=v \cdot|p-q|$, where $v=\Theta(\sqrt{k}) \cdot|p-q|$ if $k \leq 5(p-q)^{-2}$ and $v \geq 1-\exp \left(-(p-q)^{2} k / 3\right)$ otherwise.

The (rather technical) proof appears in the appendix.

\section{Conclusion}

The obvious way of using statistical information (e.g., a binary guess that is positively correlated with the correct value) is to amplify the confidence level of the information and use it as if it were certainly correct. The current work studies an alternative method of using statistical information and shows that in some settings using unreliable information directly works quite well. This was demonstrated already in Section 1.2, whereas the rest of this work studies the question of how to make the best use of multiple independent copies of such statistical information.

\section{Acknowledgments}

We are grateful to Ofer Zeitouni and Dana Ron for useful discussions. 


\section{References}

[1] W. Feller. An Introduction to Probability Theory and Its Applications, Volume II. John Wiley \& Sons, 2nd ed., 1972.

[2] O. Goldreich. Foundation of Cryptography - Basic Tools. Cambridge University Press, 2001.

[3] O. Goldreich. Foundation of Cryptography - Basic Applications. Cambridge University Press, 2004.

\section{Appendix: Proof of Proposition 2.5}

We shall approximate $\mathcal{V}_{(p, q)}\left(f_{\text {sd }}^{(k)}\right)$ by using Eq. (16) (rather than Eq. (21)). Recall that by Eq. (16) we have

$$
\mathcal{V}_{(p, q)}\left(f_{\mathrm{sd}}^{(k)}\right)=(p-q) \cdot \mathrm{E}\left[\operatorname{sign}\left(S_{k}\right)\right]
$$

where $S_{k}=\sum_{i=1}^{k} T_{i}$ (and $T_{i}=X_{i}-Y_{i}$ ). We assume, without loss of generality, that $p>q$ and lower bound the value of $\mathrm{E}\left[\operatorname{sign}\left(S_{k}\right)\right]$, using $\mathrm{E}\left[T_{i}\right]=p-q$. We distinguish three cases according to the relation between $k$ and $p-q$ :

Case 1: $k \geq 5(p-q)^{-2}$. In this case we use the (standard additive) Chernoff Bound, and derive

$$
\begin{aligned}
\mathrm{E}\left[\operatorname{sign}\left(S_{k}\right)\right] & =\operatorname{Pr}\left[S_{k}>0\right]-\operatorname{Pr}\left[S_{k}<0\right] \\
& >1-2 \cdot \operatorname{Pr}\left[S_{k} \leq 0\right] \\
& >1-2 \cdot \exp \left(-\frac{(p-q)^{2} \cdot k}{2}\right) .
\end{aligned}
$$

This establishes the relevant part of the claim (i.e., $\mathcal{V}_{(p, q)}\left(f_{\mathbf{s d}}^{(k)}\right)=v \cdot|p-q|$, where $v=$ $\left.1-2 \exp \left(-(p-q)^{2} k / 2\right)>1-\exp \left(-(p-q)^{2} k / 3\right)\right)$.

The following complemantary two cases are distinguished according to a constant $c \geq 5$ that depends only on $\gamma_{p, q} \stackrel{\text { def }}{=} \sqrt{p(1-p)+q(1-q)}$.

Case 2: $k \in\left[c \cdot(p-q)^{-1}, 5(p-q)^{-2}\right]$. In this case we use the Berry-Esseen estimate of the Central Limit Theorem (cf., e.g., [1, Sec. XVI.5]). Specifically, we approximate E[ $\left.\operatorname{sign}\left(S_{k}\right)\right]$ by $\mathrm{E}\left[\operatorname{sign}\left(\widetilde{S}_{k}\right)\right]$, where $\widetilde{S}_{k}$ is the normal distribution approximation of $S_{k}$; that is,

$$
\widetilde{S}_{k} \stackrel{\text { def }}{=} k \cdot(p-q)+\sqrt{k} \cdot \gamma_{p, q} \cdot \mathrm{N}(0,1)
$$

where $\mathrm{N}(0,1)$ denotes the normal distribution (with mean 0 and variance 1 ), and $\sqrt{k} \cdot \gamma_{p, q}$ replaces $\sqrt{\operatorname{Var}\left[S_{k}\right]}=\sqrt{k} \cdot \sqrt{(p(1-p)+q(1-q))}$. More formally, we use the fact that for every $r$ it holds that that

$$
\left|\operatorname{Pr}\left[S_{k}>r\right]-\operatorname{Pr}\left[\widetilde{S}_{k}>r\right]\right|<\epsilon \stackrel{\text { def }}{=} \frac{3 \rho}{\gamma_{p, q}{ }^{3} \sqrt{k}}
$$


where $\rho=\mathrm{E}\left[\left|T_{1}-(p-q)\right|^{3}\right]<2 \cdot \gamma_{p, q}{ }^{2}$. It follows that

$$
\begin{aligned}
\mathrm{E}\left[\operatorname{sign}\left(S_{k}\right)\right] & =\operatorname{Pr}\left[S_{k}>0\right]-\operatorname{Pr}\left[S_{k}<0\right] \\
& =\operatorname{Pr}\left[\widetilde{S}_{k}>0\right]-\operatorname{Pr}\left[\widetilde{S}_{k}<0\right] \pm 2 \epsilon \\
& =2 \operatorname{Pr}\left[\widetilde{S}_{k}>0\right]-1 \pm 2 \epsilon .
\end{aligned}
$$

Now, we analyze $\operatorname{Pr}\left[\widetilde{S}_{k}>0\right]$ via

$$
\operatorname{Pr}\left[(p-q) k+\sqrt{k} \gamma_{p, q} \cdot \mathrm{N}(0,1)>0\right]=\operatorname{Pr}\left[\mathrm{N}(0,1)>-\frac{p-q}{\gamma_{p, q}} \cdot \sqrt{k}\right]
$$

Setting $r \stackrel{\text { def }}{=}(p-q) \sqrt{k} \leq 1$, it follows that $\operatorname{Pr}\left[\mathrm{N}(0,1)>-r / \gamma_{p, q}\right]=0.5+\Theta(r)$. So Eq. (27) yields $\Theta(\sqrt{k} \cdot(p-q))-\Theta\left(k^{-1 / 2}\right)$, which is lower bounded by $\Theta(\sqrt{k} \cdot(p-q))$, when using $k \geq c \cdot(p-q)^{-1}$ (where $c$ is large enough w.r.t the above hidden constants). It follows $\mathcal{V}_{(p, q)}\left(f_{\mathrm{sd}}^{(k)}\right)=\Theta(\sqrt{k}) \cdot(p-q)^{2}$, which establishes the other part of the claim for the current case.

Case 3: $k \leq c \cdot(p-q)^{-1}$. It suffices to establish that $\mathcal{V}_{(p, q)}\left(f_{\mathrm{sd}}^{(k)}\right)=\Theta(\sqrt{k}) \cdot(p-q)^{2}$, for $k \leq$ $(p-q)^{-1}$. This is done by writing $T_{i}$ as $T_{i}^{\prime}+\left(1-T_{i}^{\prime}\right) \cdot T_{i}^{\prime \prime}$, where $T_{i}^{\prime} \in\{0,1\}$ and $T_{i}^{\prime \prime} \in\{-1,0,1\}$ are independent random variables satisfying $\operatorname{Pr}\left[T_{i}^{\prime}=1\right]=p-q$ and $\operatorname{Pr}\left[T_{i}^{\prime \prime}=1\right]=\operatorname{Pr}\left[T_{i}^{\prime \prime}=\right.$ $-1]=\frac{q-p q}{1-(p-q)}$. Letting $S_{k}^{\prime}=\sum_{i \in[k]} T_{i}^{\prime}$ and $S_{k}^{\prime \prime}=\sum_{i \in[k]} T_{i}^{\prime \prime}$, we have

$$
\begin{aligned}
\mathrm{E}\left[\operatorname{sign}\left(S_{k}\right)\right] & =\sum_{j=0}^{k} \operatorname{Pr}\left[S_{k}^{\prime}=j\right] \cdot \mathrm{E}\left[\operatorname{sign}\left(S_{k-j}^{\prime \prime}+j\right)\right] \\
& =\sum_{j=0}^{k} \operatorname{Pr}\left[S_{k}^{\prime}=j\right] \cdot\left(\mathrm{E}\left[\operatorname{sign}\left(S_{k-j}^{\prime \prime}\right)\right]+2 \cdot \operatorname{Pr}\left[0 \leq S_{k-j}^{\prime \prime}<j\right]\right)
\end{aligned}
$$

where $S_{k-j}^{\prime \prime}$ represents the sum of the $k-j$ variables $T_{i}^{\prime \prime}$ that correspond to the indices $i$ that satisfy $T_{i}^{\prime}=0$ (i.e., $S_{k-j}^{\prime \prime}$ represents $\sum_{i \in I} T_{i}^{\prime \prime}$, where $I=\left\{i: T_{i}^{\prime}=0\right\}$ ). Since $\mathrm{E}\left[\operatorname{sign}\left(S_{k-j}^{\prime \prime}\right)\right]=0$ (becuase $\mathrm{E}\left[T_{i}^{\prime \prime}\right]=0$ ), Eq. (30) simplifies to

$$
2 \cdot \sum_{j=1}^{k} \operatorname{Pr}\left[S_{k}^{\prime}=j\right] \cdot \operatorname{Pr}\left[0 \leq S_{k-j}^{\prime \prime}<j\right] .
$$

The lower bound in the claim (i.e., $v=\Omega(\sqrt{k} \cdot(p-q))$ ) follows once we prove that $\operatorname{Pr}\left[S_{k}^{\prime}=\right.$ $1] \cdot \operatorname{Pr}\left[S_{k-1}^{\prime \prime}=0\right]=\Omega(\sqrt{k} \cdot(p-q))$. We start by noting that

$$
\begin{aligned}
\operatorname{Pr}\left[S_{k}^{\prime}=1\right] \cdot \operatorname{Pr}\left[S_{k-1}^{\prime \prime}=0\right] & =k \cdot(p-q)(1-(p-q))^{k-1} \cdot \operatorname{Pr}\left[S_{k-1}^{\prime \prime}=0\right] \\
& >\frac{(p-q) k}{3} \cdot \operatorname{Pr}\left[S_{k-1}^{\prime \prime}=0\right]
\end{aligned}
$$

In order to estimate $\operatorname{Pr}\left[S_{k-1}^{\prime \prime}=0\right]$, we write $S_{k-1}^{\prime \prime}$ as the difference of $\sum_{i \in[k-1]} X_{i}^{\prime \prime}$ and $\sum_{i \in[k-1]} Y_{i}^{\prime \prime}$, where the $X_{i}^{\prime \prime}$ 's and $Y_{i}^{\prime \prime \prime}$ 's are iid $0-1$ random valiables (i.e., $p^{\prime \prime}=\operatorname{Pr}\left[X_{i}^{\prime \prime}=1\right]$ satisfies $\left.p^{\prime \prime}\left(1-p^{\prime \prime}\right)=\frac{(1-p) q}{1-(p-q)}\right)$. We get

$$
\operatorname{Pr}\left[S_{k-1}^{\prime \prime}=0\right] \geq \sum_{j=(k-1) p^{\prime \prime} \pm \sqrt{k-1}} \operatorname{Pr}\left[\sum_{i \in[k-1]} X_{i}^{\prime \prime}=j\right] \cdot \operatorname{Pr}\left[\sum_{i \in[k-1]} Y_{i}^{\prime \prime}=j\right]
$$




$$
\begin{aligned}
& =\sum_{j=(k-1) p^{\prime \prime} \pm \sqrt{k-1}} \operatorname{Pr}\left[\sum_{i \in[k-1]} X_{i}^{\prime \prime}=j\right]^{2} \\
& >\frac{\operatorname{Pr}\left[\sum_{i \in[k-1]} X_{i}^{\prime \prime}=(k-1) p^{\prime \prime} \pm \sqrt{k-1}\right]^{2}}{2 \sqrt{k-1}+1} \\
& >\frac{\operatorname{Pr}\left[\sqrt{(k-1) \gamma_{p^{\prime \prime}, p^{\prime \prime}}} \cdot \mathrm{N}(0,1)= \pm \sqrt{k-1}\right]^{2}-o(1)}{2 \sqrt{k-1}+1}
\end{aligned}
$$

where the last inequality uses the Berry-Esseen estimate of the Central Limit Theorem. Observing that $\operatorname{Pr}\left[\mathrm{N}(0,1)= \pm 1 / \gamma_{p^{\prime \prime}, p^{\prime \prime}}\right]=\Omega(1)$, it follows that $\operatorname{Pr}\left[S_{k-1}^{\prime \prime}=0\right]=\Omega(1 / \sqrt{k-1})$, and so Eq. (32) is $\Omega((p-q) k / \sqrt{k-1})$ (and the same holds w.r.t Eq. (31)). To upper bound Eq. (31), we note that it can be upper bounded by

$$
\begin{aligned}
2 \cdot \sum_{j=1}^{k} \operatorname{Pr}\left[S_{k}^{\prime}=j\right] \cdot j \cdot \operatorname{Pr}\left[S_{k-j}^{\prime \prime}=0\right] & <2 \cdot \sum_{j=1}^{k}\left(\begin{array}{l}
k \\
j
\end{array}\right) \cdot(p-q)^{j} \cdot j \cdot \operatorname{Pr}\left[S_{k-j}^{\prime \prime}=0\right] \\
& =O\left((p-q) k \cdot \operatorname{Pr}\left[S_{k-1}^{\prime \prime}=0\right]\right)
\end{aligned}
$$

and the claim follows because $\operatorname{Pr}\left[S_{k-1}^{\prime \prime}=0\right]=O(1 / \sqrt{k})$. This establishes $\mathcal{V}_{(p, q)}\left(f_{\text {sd }}^{(k)}\right)=$ $\Theta(\sqrt{k}) \cdot(p-q)^{2}$ also in the current case.

The proposition follows. 